\title{
UNIDIRECTIONAL MOVEMENT FIBRES FROM A PROPRIOCEPTIVE ORGAN OF THE CRAB, CARCINUS MAENAS
}

\author{
By C. A. G. WIERSMA* \\ (California Institute of Technology) \\ AND E. G. BOETTIGER* \\ (University of Connecticut) \\ From the Zoological Laboratory, Cambridge
}

(Received I4 fuly 1958)

Proprioception in the legs of arthropods arises in receptor ceils more closely associated with structures of the joints than with muscles. Sensitive endings are located in special internally placed organs (Barth, 1934; Burke, 1954; Alexandrowicz \& Whitear, 1957). Studies of the action potentials in the leg nerve during mechanical manipulation of the leg joints in Limulus (Pringle, 1956) and in certain arachnids (Pringle, 1955) have shown the presence of several types of proprioceptive endings. Slow-adapting tonic position fibres, some responding at the flexed and others at the extended positions, were shown to have their endings situated so as to react to strains in the cuticle. The rapidly adapting phasic fibres, however, originate in some internal structures, different fibres responding to the two directions of movement.

Burke (1954) described and studied an internal organ present in the joint between the dactylopodite and propodite of Carcinus maenas, the shore crab. Phasic responses were initiated during movement in either direction, and resting discharges continued when the claw was held in the open or closed positions. This organ, the pro-dactylopodite (P.D.) organ, consists of a stretched elastic strand and associated nerve cells. It is attached at one end to the inner surface of the dactylopodite and at the other to an apodeme, functioning as the tendon for the closer muscle. As this tendon is moved by the muscle during closing (flexion), the elastic strand is further stretched, increasing its length by about $\mathrm{I} \mathrm{mm}$. As this structure signals both position and movement, it is important to know how many different types of endings are present: and also whether a single organ with such an apparently simple structure can completely analyse both directions of movement, and in addition signal the degree of both flexion and extension. The single-fibre technique was therefore employed to determine the types of sensory axons making up the P.D. nerve bundle of Carcinus.

- John Simon Guggenheim Memorial Fellow. 


\section{METHODS}

In most experiments the organ was left in situ in order to avoid the changes in excitability resulting from interference with its suspensions when dissections are performed near it. The experimental set-up employed is illustrated in Fig. I. The walking legs of Carcinus maenas were used throughout. The nerve was exposed in the meropodite by removing the inner half of the surrounding shell and all the muscles. The nerve was first split with fine needles into the naturally occurring bundles. Bundles not reacting to manipulation of the joint were discarded, whereas any active bundle was kept. In this way a single thin naturally occurring bundle

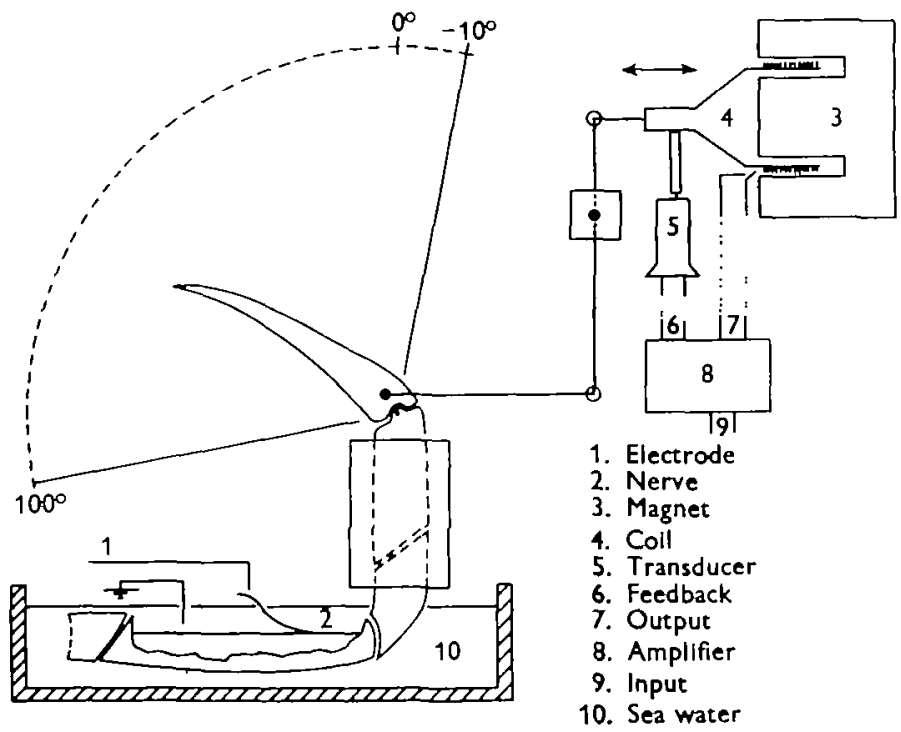

Fig. I. Drawing showing preparation and experimental apparatus.

was regularly found, and subsequently further divided by the technique previously described for obtaining single active efferent fibres (Wiersma, I94I). Carcinus proved to be highly suitable for this purpose, as splitting of the fine subbundles could be more readily performed than in most other species. Splitting in either direction was usually successful. In most other species, unless splits are made from the periphery, connective tissue strands are liable to constrict the fibres, in Carcinus these strands are weak. Larger fibres could be used singly, but small ones have so much electrical resistance and dry out so quickly that in isolation they are not suitable for the leading off methods employed. Instead, small bundles of such fibres were prepared and used if the few reacting fibres in them gave response patterns which could be distinguished with certainty.

The nerve was kept just below the level of the sea water in which the preparation was submerged. After a small bundle was obtained in which the polyphasic lead showed the presence of a promising fibre, the lead was made monophasic by lifting the little bundle out of the fluid with the active electrode and letting it dry out. The 
other electrode remained in the water and was connected to earth. Under these circumstances, the place where the bundle breaks through the surface acts as the active electrode. The action potential spikes were amplified by a Tectronic I I 2 preamplifier, the output of this being fed into a Cossor oscilloscope and a loud speaker.

The receptor organ was mechanically stimulated by moving the dactylopodite passively by means of the coil of a permanent magnetic loudspeaker unit, which functioned as an element of a servomechanism activated by voltage changes. The response of the unit was very rapid and non-oscillatory. Extremely smooth movements were achieved by feedback control of the heavily damped coil. Ordinarily, movements with a constant velocity were used in this investigation. These were produced by obtaining the activating voltage change from a Hewlett-Packard function-generator capable of producing linear voltage changes which caused less than $\mathrm{a}_{1} \% \mathrm{sec}$. movement of the dactylopodite.

The actual movement of the coil, picked up by a transducer which furnished the negative feedback, was also used to drive the second beam of the oscilloscope. The movement recorded on the films was therefore that of the coil and not of the dactylopodite. The latter's movement followed that of the coil very closely, in spite of a slight unavoidable play in the lever system linking them and of the small error introduced by converting linear to angular motion.

\section{RESULTS}

Confirming Burke's findings (1954), a specific bundle of large and small fibres responsive to manipulation of the dactylopodite could readily be isolated in the meropodite. This bundle, usually located adjacent to the closer motor axons, proved to contain all the fibres giving proprioceptive discharges from the P.D. organ. In some instances responses to movement were found in other bundles, but on closer observation these were shown to arise from different sources. Among these were hairs on the dactylopodite and propodite, and proprioceptors of other joints of the leg, which come readily into play unless special precautions are taken. Using a large clamp and moving the dactylopodite by means of a pin inserted into its base, stimulation of other receptors could be avoided. Under these conditions, all fibres reacting to displacement of the P.D. joint were found to be restricted to the P.D. bundle. As will be shown later, not all fibres in the P.D. bundle arise from the P.D. organ itself, but such fibres did not play a part in the responses to be described (see below).

\section{DESCRIPTION OF THE FIBRE RESPONSES}

The unit responses obtained show clearly that the reacting sensory cells can be divided into a number of response types. In the first place, about half of the fibres respond to opening (extension of the dactylopodite), whereas the others respond to closing (flexion). In each of these classes, fibres are present which discharge only during the movement of the dactylopodite, and others which continue to fire when the joint is near one of the extreme positions. Among the fibres responsive only 
during movements, none were found that respond in any part of the arc to both opening and closing.

A. Position fibres. Occasionally fibres are found which approach closely the ideal responsiveness of a structure designed solely for signalling position. Such a receptor should have no adaptation and should be unaffected by movement. Fig. $2 a$ illustrates the responses of two such fibres present in a small bundle. On moving the dactylopodite from -5 to $-10^{\circ}$ (see Fig. I) there is no burst of impulses in either fibre during movement, but in both the frequency increased markedly with changing position and was maintained during the remainder of the record. Indeed, such fibres will continue to fire, with little change in frequency, for very long periods. As with many fibres of this type, these two are sensitive to position over only a small part of the total arc. They both stopped discharging at $o^{\circ}$.

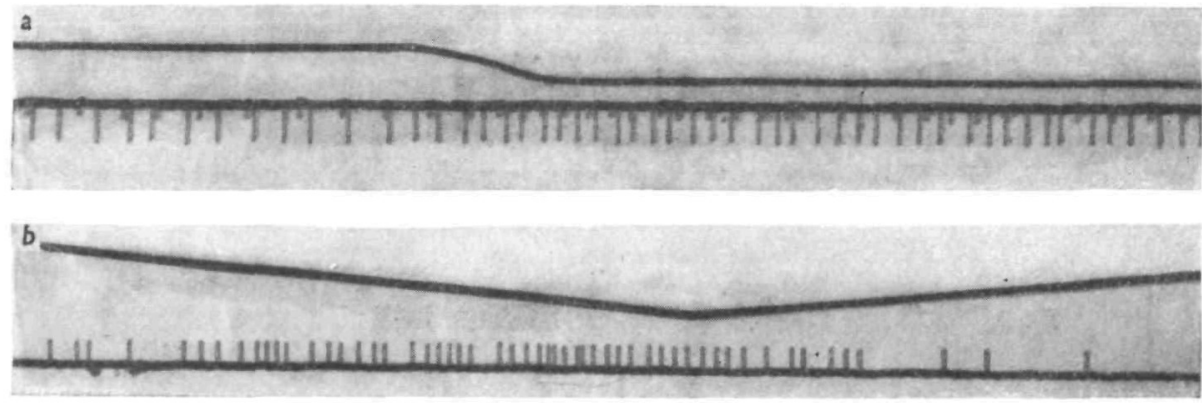

Fig. 2. Position fibres. Upper line indicates movement of the dactylus, down opening; up closing. Lower line shows action potentials. (a) 'Pure' position fibre; (b) position fibre showing different sensitivity at the same position attained during opening and closing.

Commonly encountered are fibres in which adaptation is more pronounced, though a tonic discharge is maintained in the most extreme position. These are usually larger than the more ideal type and so are easier to find. In Fig. $2 b$ the response of such a fibre is shown when the dactylopodite was moved toward and away from the extreme opener position. The fibre starts to discharge at $10^{\circ}$ but stops on the return at $5^{\circ}$. The whole discharge is markedly asymmetrical, there being many more impulses during opening than during closing. The behaviour of this type of fibre does not need any special discussion, since it is similar to that of the ligament organ in vertebrates (Boyd \& Roberts, 1953).

Except for those signalling the more extreme positions, position fibres are difficult to prepare by the methods employed. It is therefore uncertain whether the complete range of position is covered. Most position fibres are of small diameter, and failure to find them may be due either to their size or to their rarity. Certain fibres, responding tonically to one of the extreme positions, are also brought into activity by rapid movement and are discussed below.

B. Unidirectional movement fibres. Some of these fibres, which are encountered in every preparation, discharge on the slightest movement, provided it is in the 
direction for which the ending is sensitive. The threshold of the most sensitive opening movement fibres is lower than for the most sensitive closing fibres. Sensitive opening movement fibres begin to fire at movement velocities of less than $\mathrm{I}^{\circ} / \mathrm{sec}$., at which speed it takes $2 \mathrm{~min}$. to open fully from the closed position $\left(110^{\circ}\right.$,
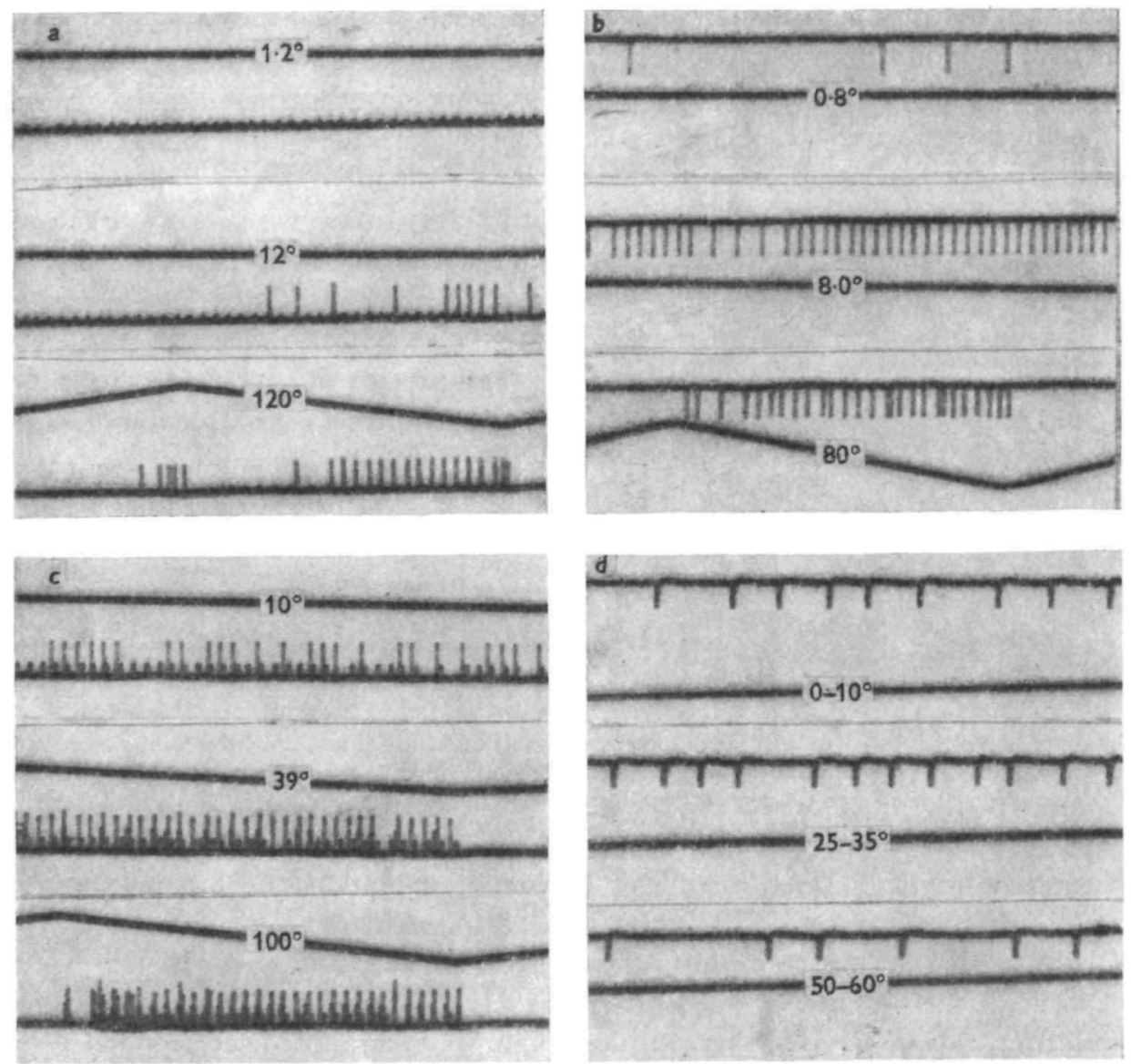

Fig. 3. Movement fibres. Upper line indicates movement of the dactylopodite, down opening; up closing. Lower line shows action potentials. In $(a),(b)$ and $(c)$ the figures on the records show the speed of movement in degrees per sec. In $(d)$ the figures give the movement position arc in degrees, the velocity being constant at $1 \% \mathrm{sec}$. (a) A very sensitive opening fibre, a less sensitive opening fibre, and a very insensitive closing fibre firing only at the end of the movement. (b) A sensitive opening fibre showing double firing at the fastest speed. (c) Three opening fibres with different sensitivities. ( $d$ ) A sensitive closing fibre showing response at different portions of the movement arc at near threshold velocity.

see Fig. 3). Their responses are very nearly those of a pure unidirectional movement fibre, differing from the ideal, near threshold and at high velocities, in two ways. In the first place, at threshold they are not completely independent of position. In general, the opening fibres are less sensitive to movement near the closed position 
and the closing fibres near the opened position. However, for the rest of the movement they are as responsive in one part of the arc as in another. Secondly, the movement fibres are not quite independent of the velocity of movement. Near threshold speeds the spikes appear very irregularly, but while the movement velocity is still very low a maximum frequency is attained over the whole arc. Thus in Fig. $3^{b}$ there is no significant increase in frequency though the speed increases from 8 to $80^{\circ} / \mathrm{sec}$. The small fibre shown in Fig. $3 a$ was one of the most sensitive found. It attained full saturation, about $40 \mathrm{impulses} / \mathrm{sec}$., at $\mathrm{I} \cdot 2 \% \mathrm{sec}$. and showed no change in frequency to $120 \%$ sec. (complete opening movement in $\mathrm{r} \mathrm{sec}$.). Even at speeds three or four times as fast, the same signal could be detected without change in frequency, though partially obscured by the signals of the other fibres. Such fibres cannot play a significant role in mediating the velocity of movement, but only in mediating the movement itself and its direction.

In Fig. $3 d$ the response of a sensitive closing movement fibre is shown near threshold, the three records being from different parts of the movement arc as indicated. Some increase in frequency is evident near the mid-position and the firing is quite irregular. The response of a small bundle of opening movement fibres is shown in Fig. $3 c$. The smallest response is that of the most sensitive fibre, which is saturated at the slowest speed shown. The largest response is of a fibre with a higher threshold, which is not fully saturated at this speed but becomes so at $39 \% \mathrm{sec}$. The fibre with intermediate spike height is the most insensitive and does not fire at the slowest speed. At $39^{\circ} / \mathrm{sec}$. it responds in an irregular manner and by $100 \% \mathrm{sec}$. fires regularly. In all these fibres, though they have such very different thresholds, the range of velocities through which the discharge frequency increases is thus limited, and each by itself is a poor indicator for velocity.

Though there was no graded increase in frequency with increase in speed in any of these fibres, some have a strong tendency to give more and more double or even triple discharges at high velocities of movement. Multiple responses are illustrated in Fig. $3 b$ and $c$ at the fastest speeds.

Rather regularly encountered among the large movement fibres are some responsive at threshold speeds only to movement at one side or the other of the midposition. Occasionally one was found that fired only at the beginning of a full opening movement if the fibre was an opening fibre, or at the beginning of closure if the fibre was a closing fibre. Such behaviour is difficult to explain and may not be normal. Usually opening fibres of this type are most sensitive near the opened position and closing fibres near the closed position. Frequency of discharge is more dependent on both velocity and the degree of opening (or closing) than in the sensitive movement fibres. Such an opening fibre will have a lower threshold near the opened position, and the further from the opened position the greater will be the movement speed required to produce any given discharge frequency. Toward the closure side the fibre may not fire at the fastest speeds used, though certainly some can be made to respond in any region. The large spikes in Fig. $3^{a}$ are from two different fibres, an opening and a closing, firing only after the movement has passed the mid-position. At $12 \%$ sec. the opening fibre begins to fire and at $120^{\circ} / \mathrm{sec}$. its 
discharge has become regular but limited to about one-half of the arc. The closing fibre shows only five spikes at the end of closure at $120 \%$ sec. Fibres with the larger spikes tend to adapt to the movement stimulus more readily and the largest ones may show a greater increase in frequency than those illustrated; but such fibres were rarely found.

There appears to be a complete gradation from fibres responding as nearly pure movement fibres, affected little by velocity or position, to those whose discharge frequency is affected by both to various degrees. Fibres of the latter type are commonly used by vertebrates for signalling proprioceptive information, while specialization toward the development of pure unidirectional movement fibres has not previously been reported.

Some fibres behave as rapidly adapting unidirectional movement fibres over part or all of the movement arc, and as position fibres when the dactylopodite is at the end of its movement, fully opened for opener fibres and fully closed for closure fibres. This type has been found especially in other species of Crustacea (Wiersma, 1959). With respect to the classification used here these fibres are intermediate.

\section{LOCALIZATION OF CELLS RESPONSIBLE FOR DIFFERENT FIBRE RESPONSES}

Preparations of the P.D. organ were made by opening the propodite on the lateral posterior side. By removing the muscle fibres of the closer muscle on that side, the organ can be readily exposed with the nerve bundle going to it. These preparations were used for physiological experimentation and subsequent staining with Rongalit methylene blue (see Fig. 4). On the whole, our results were similar to those of Burke with respect to the anatomical relations.
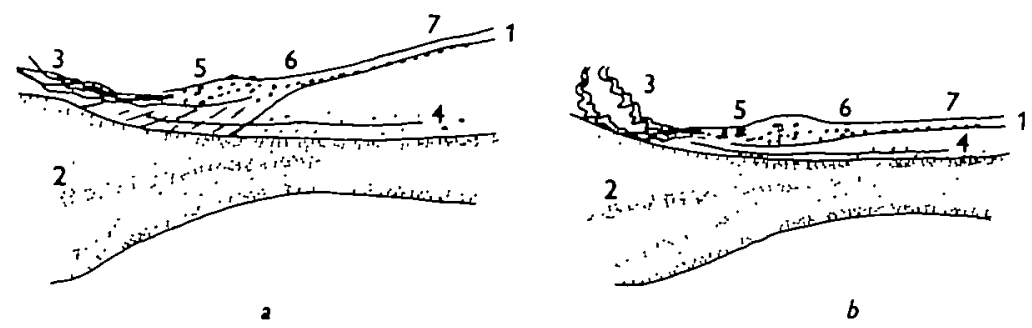

Fig. 4. Drawing of the P.D. organ showing the orientation of the elastic strand and the positions of the cells referred to in the text. (1) Elastic strand. (2) Tendon. (3) P.D. bundle. (4) Tendon nerve branch. (5) Proximal cell group. (6) Distal cell group. (7) Line of small cells extending up the strand. (a) Opened position; (b) closed position.

Large cells are clustered at the tendon end of the organ and small cells extend along a good part of the elastic strand. It was found that a branch of the nerve bundle leading to the organ leaves the main part of the bundle to go to the distal part of the tendon. In some cases, one or two nerve cells could be discerned, but the further course of the fibres was not revealed. 
The branch can be split off rather readily from the main bundle, and three types of experiments were performed in order to establish the role, if any, that this branch plays in the response on movement of the dactylopodite. (a) In preparations in which the main bundle gave clear responses of all types the tendon branch was invariably silent. $(b)$ Cutting the tendon branch between a point near the closer tendon and its junction with the point where it joins the main bundle did not alter the response obtained from the P.D. bundle in any noticeable way. More specifically, all four main types of responses were still present. (c) Cutting the branches going to the P.D. organ, but leaving the tendon branch, resulted in every instance in the disappearance of all responses in the P.D. bundle to manipulation of the joint. As to the kind of stimulus mediated by the tendon branch we have no experimental evidence.

In the main bundle one can readily detect responses of different kinds. But the exposed organ is much more sensitive to outside disturbances such as water movements. Hence it is not possible to distinguish the responses with the same precision as in the meropodite preparation. The reason for using this preparation was that it is possible to split the bundle into several subbundles which can be traced to their origin. Especially in the closed position, the nerve near P.D. is coiled and can be seen to consist of several bundles (see Fig. $4 b$ ). It was found possible to split these naturally occurring divisions further until enough length of nerve for leading-off purposes was obtained.

There are often two main subbundles each coming from a different side of the organ. A successful split brought about the separation of opening and closing fibres. The bundle containing the closing fibres showed often a peripheral and a central branch. A further split revealed that the peripheral branch contained many position fibres, the central one preponderantly movement fibres. For the other subbundle a similar division of opening movement fibres and opening position fibres was attained rather clearly in a single experiment only. Further evidence is necessary to establish this localization of function.

By tracing the subbundles which reacted predominantly to one type of stimulation it was found that opening fibres originated from a cluster of cells, some large and others small, located near the tendon end of the organ but rather on the side away from the tendon (see Fig. 4(5)). The closing subbundle was traced to another cluster not quite so loosely attached to the elastic strand and situated somewhat further distal and more on the tendon side (see Fig. 4(6)). As stated, when a peripheral bundle could be prepared it contained position fibres and could be traced to the small cells which extend along the elastic strand towards its insertion on the dactylopodite (see Fig. 4(7)).

\section{DISCUSSION}

In view of the structure of the P.D. organ, the most surprising result of this study is the strictly unidirectional nature of the individual endings of the different sense cells, whereas the organ as a whole has similar sensitivity for both directions. The separation of the endings sensitive to flexion from those sensitive to extension must 
be related to the mechanical stresses to which the two types of endings are subjected. How this can be brought about is therefore an important question to which there is, as yet, no certain answer. As was shown by Burke (1954), the elastic strand in which the nerve endings appear to be embedded is under tension in all positions of the dactylopodite. It can be observed that, on closing, the tendon moves in such a direction as to increase the tension in the elastic strand. At the same time the angle between the tendon and the elastic strand decreases from 17 to $7^{\circ}$, a change of $10^{\circ}$, due partly to the movement of the tendon and partly to rotation of the dactylopodite which moves the insertion of the elastic strand (see Fig. 4). The presence of linear as well as angular movements-which are of opposite phase, the angle being smallest when the linear change is largest--suggests a possible explanation. Many of the fibres of the elastic strand attach to the tendon at points which are located as a straight continuation of the strand itself. But others, rather thin and more difficult to see, run to the tendon across the angle between the strand and the tendon. During closure, when tension in the main strand increases, the tension in the latter elastic fibres would decrease, since the angle is reduced. One would, therefore, expect to find that the group of nerve cells shown to be associated with opening have their endings associated with the elastic fibres fanning out from the strand towards the tendon, whereas the group of closure cells should be more closely associated with the main strand of elastic fibres.

In our methylene-blue stained preparations no final decision on this important point could be reached. The opening movement cells were closer to the point where the strand attaches to the tendon and were rather loosely attached to it. However, they lie generally more on the side of the organ away from the fanning elastic fibres. Certain stained preparations were photographed with the organ in extreme opened and closed positions (see Fig. 4a,b). In these it could be seen that the cell positions alter considerably, showing that indeed different cell groups are exposed to different displacements and forces. It will be shown in another paper (Wiersma, 1959) that in other joint organs the stimulation of most of the sense cells comes about when the tension in the main elastic strand is diminished. Further studies are required to show the relations between the nerve endings and the elastic fibres, and what constitutes the stimulating factor. Although the nerve endings were not studied in detail it was noted that the dendrites of the large bipolar cells at the proximal end of the organ appeared as spirals.

It has been pointed out that individual movement fibres are usually poor velocity fibres. By the recruitment of several fibres with different sensitivities during faster movements the C.N.S. could, of course, extract this information. When the different types of position fibres are also considered it is evident that enough information is transmitted to allow a very precise analysis of any peripheral change in the joint, the only possible exception being that there is no information around the midposition when the joint is kept perfectly still. In contrast with Burke, we observed a number of instances in which no fibres were observed to fire under these conditions, but the possibility that very small fibres would regularly do so is not completely excluded. 
From our observations, Burke's thesis that the organ is used to detect vibrations appears unlikely. We have confirmed his observation that the isolated organ is very sensitive to vibrations, but have found that the responses to movements as small as he used occurred in the most sensitive unidirectional movement fibres, and in these only during the appropriate phase of the movement. Consequently, these fibres could not distinguish between a vibration and a continuous movement. In experiments in which the dactylopodite was vibrated at various amplitudes up to IOO cyc./sec. the very small movements were always detected only in the sensitive movement fibres. As the amplitude was increased, velocity increasing as well, the larger rapidly adapting fibres responded during the phase of the movement appropriate to their sensitivity. In these fibres one-to-one responses were found up to roo cyc./sec., whereas the sensitive fibres do not usually discharge above $4^{\circ}$ impulses/sec.

There seems little doubt that the development of sensory endings mediating unidirectional movement nearly independent of position or velocity, is connected with the presence of an exoskeleton and the absence of sense organs in the muscles. In animals with an internal skeleton similar endings could occur in the joints. It is interesting to note that movement fibres have been reported in the knee joint of the cat, though these are not unidirectional (Boyd \& Roberts, 1953).

The authors wish to express their appreciation to members of the staff of the Zoological Laboratory, Cambridge, for providing facilities and equipment necessary for this investigation and for inviting us to work in the Zoological Laboratory during the tenure of our Fellowships.

\section{SUMMARY}

I. The proprioceptive organ of the shore crab Carcinus maenas, which signals all proprioceptive sensation from the joint between propodite and dactylopodite, has been shown to contain sense cells receptive to position as well as to movement.

2. Certain cells of the organ increase their discharge frequency in response to greater degrees of flexion, while other endings respond to greater degrees of extension. Movement has little effect on the frequency of the position fibres.

3. Cells responding to movement in one direction only are present, certain ones signalling flexion and others extension. The most sensitive undirectional movement fibres are almost completely independent of position and velocity. They provide the crab with an extremely sensitive movement sense not as yet reported in other proprioceptive mechanisms.

4. Both position-sensitive and movement-sensitive cells show a wide range of thresholds.

5. Cells which cannot be strictly classified as either position or movement sensitive have also been found.

6. By tracing the origin of the signals to the cell bodies, it appears that movement fibres have on the whole larger cell bodies than position fibres and are more proximally located. 
iz C. A. G. Wiersma and E. G. Boettiger

7. Flexor and extensor movement cells appear to lie on different sides of elastic strand.

8. The question of how unidirectional sensitivity may be achieved is discussed.

\section{REFERENCES}

Alexandrowicz, J. S. \& Whitear, M. (1957). Receptor elements in the coxal region of decapoda crustacen. F. Mar. Biol. Ass. U.K. 36, 603-28.

BARTH, G. (1934). Untersuchungen über Myochordotonalorgane bei dekapoden Crustaceen. Z. wiss. Zool. 145, 576-624.

Boyd, I. A. \& RoberTs, T. D. M. (1953). Proprioceptive discharges from stretch-receptors in the knee-joint of the cat. 7. Physiol. 122, 38-58.

BURKk, W. (1954). An organ for proprioception and vibration sense in Carcinus maenas. I. Exp. Biol. 31, 127-38.

Pringle, J. W. S. (I955). The function of the lyriform organs of arachnids. 7. Exp. Biol. 32, 270-8.

Princle, J. W. S. (1956). Proprioception in Limulus. F. Exp. Biol. 33, 658-67.

Wiersma, C. A. G. (I94r). The inhibitory nerve supply of the leg muscles of different decapod crustaceans. F. comp. Neurol. 74, 63-79.

Wiersma, C. A. G. (1959). Movement reception in decapod Crustacea (in preparation) (for F. Mar. Biol. Ass. U.K.). 